DOI: $10.2478 / \mathrm{v} 10324-012-0018-\mathrm{z}$<smiles>CC1=CC=C1</smiles>

VERSITA
Analele Universităţii de Vest,

Timişoara

Seria Matematică - Informatică

L, 2, (2012), 97- 115

\title{
Sharp Maximal Function Estimates and Boundedness for Commutator Related to Generalized Fractional Integral Operator
}

Guo Sheng, Huang Chuangxia, and Liu Lanzhe

\begin{abstract}
.
In this paper, we prove some sharp maximal function estimates for the commutators related to certain generalized fractional integral operator with general kernel and the $B M O$ and Lipschitz functions. As an application, we obtain the boundedness of the commutators on Lebesgue, Morrey and Triebel-Lizorkin spaces. The operator includes fractional Littlewood-Paley operators, Marcinkiewicz operators and Bochner-Riesz operator.
\end{abstract}

AMS Subject Classification (2000). 42B20, 42B25.

Keywords. Commutator; Littlewood-Paley operator; Marcinkiewicz operator; Bochner-Riesz operator; Sharp maximal function; Morrey space; Triebel-Lizorkin space; $B M O$; Lipschitz function.

\section{Introduction}

As the development of singular integral operators(see [9][24][25]), their commutators have been well studied(see [6][22][23]). In [8][22][23], the authors prove that the commutators generated by the singular integral operators and $B M O$ functions are bounded on $L^{p}\left(R^{n}\right)$ for $1<p<\infty$. Chanillo (see [2]) proves a similar result when singular integral operators are replaced by the 
fractional integral operators. In [3][10][19], the boundedness for the commutators generated by the singular integral operators and Lipschitz functions on Triebel-Lizorkin and $L^{p}\left(R^{n}\right)(1<p<\infty)$ spaces are obtained. In [1], some singular integral operators with general kernel are introduced, and the boundedness for the operators and their commutators generated by $B M O$ and Lipschitz functions are obtained(see [1][11]). In this paper, certain generalized fractional integral operators with general kernel are introduced, and the sharp maximal function estimates for the commutator related to the operator and the $B M O$ and Lipschitz functions are obtained, as an application, we obtain the boundedness of the commutator on Lebesgue, Morrey and Triebel-Lizorkin space. The operator includes fractional Littlewood-Paley operators, Marcinkiewicz operators and Bochner-Riesz operator.

\section{Preliminaries}

First, let us introduce some notations. Throughout this paper, $Q$ will denote a cube of $R^{n}$ with sides parallel to the axes. For any locally integrable function $f$, the sharp maximal function of $f$ is defined by

$$
M^{\#}(f)(x)=\sup _{Q \ni x} \frac{1}{|Q|} \int_{Q}\left|f(y)-f_{Q}\right| d y
$$

where, and in what follows, $f_{Q}=|Q|^{-1} \int_{Q} f(x) d x$. It is well-known that (see $[9][24])$

$$
M^{\#}(f)(x) \approx \sup _{Q \ni x} \inf _{c \in C} \frac{1}{|Q|} \int_{Q}|f(y)-c| d y .
$$

We say that $f$ belongs to $B M O\left(R^{n}\right)$ if $M^{\#}(f)$ belongs to $L^{\infty}\left(R^{n}\right)$ and define $\|f\|_{B M O}=\left\|M^{\#}(f)\right\|_{L^{\infty}}$. It has been known that (see [20])

$$
\left\|f-f_{2^{k} Q}\right\|_{B M O} \leq C k\|f\|_{B M O} .
$$

Let

$$
M(f)(x)=\sup _{Q \ni x} \frac{1}{|Q|} \int_{Q}|f(y)| d y .
$$

For $\eta>0$, let $M_{\eta}(f)(x)=M\left(|f|^{\eta}\right)^{1 / \eta}(x)$.

For $0<\eta<n$ and $1 \leq r<\infty$, set

$$
M_{\eta, r}(f)(x)=\sup _{Q \ni x}\left(\frac{1}{|Q|^{1-r \eta / n}} \int_{Q}|f(y)|^{r} d y\right)^{1 / r}
$$


Vol. L (2012) Sharp Maximal Function Estimates and Boundedness for Commutator...99

The $A_{p}$ weight is defined by (see [9])

$A_{p}=\left\{w \in L_{l o c}^{1}\left(R^{n}\right): \sup _{Q}\left(\frac{1}{|Q|} \int_{Q} w(x) d x\right)\left(\frac{1}{|Q|} \int_{Q} w(x)^{-1 /(p-1)} d x\right)^{p-1}<\infty\right\}$,

for $1<p<\infty$ and

$$
A_{1}=\left\{w \in L_{l o c}^{p}\left(R^{n}\right): M(w)(x) \leq C w(x), \text { a.e. }\right\} .
$$

For $\beta>0$ and $p>1$, let $\dot{F}_{p}^{\beta, \infty}\left(R^{n}\right)$ be the homogeneous Triebel-Lizorkin space(see [19]).

For $\beta>0$, the Lipschitz space $\operatorname{Lip}_{\beta}\left(R^{n}\right)$ is the space of functions $f$ such that

$$
\|f\|_{L i p_{\beta}}=\sup _{\substack{x, y \in R^{n} \\ x \neq y}} \frac{|f(x)-f(y)|}{|x-y|^{\beta}}<\infty .
$$

In this paper, we will study some integral operators as following(see [1]).

Definition 1. Fixed $0 \leq \delta<n$. Let $F^{\delta, t}(x, y)$ be the function defined on $R^{n} \times R^{n} \times[0,+\infty)$ and $b$ be a locally integrable function on $R^{n}$, set

$$
F^{\delta, t}(f)(x)=\int_{R^{n}} F^{\delta, t}(x, y) f(y) d y
$$

and

$$
F_{b}^{\delta, t}(f)(x)=\int_{R^{n}}(b(x)-b(y)) F^{\delta, t}(x, y) f(y) d y
$$

for every bounded and compactly supported function $f$.

Let $H$ be the Banach space $H=\{h:\|h\|<\infty\}$. For each fixed $x \in R^{n}$, we view $F^{\delta, t}(f)(x)$ and $F_{b}^{\delta, t}(f)(x)$ as the mappings from $[0,+\infty)$ to $H$. Set

$$
T^{\delta}(f)(x)=\left\|F^{\delta, t}(f)(x)\right\|,
$$

which $T_{\delta}$ is bounded on $L^{2}\left(R^{n}\right)$. The commutator related to $F^{\delta, t}$ is defined by

$$
T_{b}^{\delta}(f)(x)=\left\|F_{b}^{\delta, t}(f)(x)\right\|
$$

and $F^{\delta, t}$ satisfies: there is a sequence of positive constant numbers $\left\{C_{k}\right\}$ such that for any $k \geq 1$,

$$
\int_{2|y-z|<|x-y|}\left(|| F^{\delta, t}(x, y)-F^{\delta, t}(x, z)\|+\| F^{\delta, t}(y, x)-F^{\delta, t}(z, x) \|\right) d x \leq C,
$$

and

$$
\left(\int_{2^{k}|z-y| \leq|x-y|<2^{k+1}|z-y|}\left(|| F^{\delta, t}(x, y)-F^{\delta, t}(x, z)\|+\| F^{\delta, t}(y, x)-F^{\delta, t}(z, x) \|\right)^{q} d y\right)^{1 / q}
$$




$$
\leq C_{k}\left(2^{k}|z-y|\right)^{-n / q^{\prime}+\delta},
$$

where $1<q^{\prime}<2$ and $1 / q+1 / q^{\prime}=1$.

Definition 2. Let $\varphi$ be a positive, increasing function on $R^{+}$and there exists a constant $D>0$ such that

$$
\varphi(2 t) \leq D \varphi(t) \text { for } t \geq 0
$$

Let $f$ be a locally integrable function on $R^{n}$. Set, for $1 \leq p<\infty$,

$$
\|f\|_{L^{p, \varphi}}=\sup _{x \in R^{n}, d>0}\left(\frac{1}{\varphi(d)} \int_{Q(x, d)}|f(y)|^{p} d y\right)^{1 / p}
$$

where $Q(x, d)=\left\{y \in R^{n}:|x-y|<d\right\}$. The generalized Morrey space is defined by

$$
L^{p, \varphi}\left(R^{n}\right)=\left\{f \in L_{l o c}^{1}\left(R^{n}\right):\|f\|_{L^{p, \varphi}}<\infty\right\} .
$$

If $\varphi(d)=d^{\delta}, \delta>0$, then $L^{p, \varphi}\left(R^{n}\right)=L^{p, \delta}\left(R^{n}\right)$, which is the classical Morrey spaces (see [20][21]). If $\varphi(d)=1$, then $L^{p, \varphi}\left(R^{n}, w\right)=L^{p}\left(R^{n}\right)$, which is the Lebesgue spaces.

As the Morrey space may be considered as an extension of the Lebesgue space, it is natural and important to study the boundedness of the operator on the Morrey spaces (see [4][7][8][12][17]).

It is well known that commutators are of great interest in harmonic analysis and have been widely studied by many authors (see [22][23]). In [23], Pérez and Trujillo-Gonzalez prove a sharp estimate for the commutator. The main purpose of this paper is to prove the sharp maximal inequalities for the commutator $T_{b}^{\delta}$. As the application, we obtain the $L^{p}$-norm inequality, Morrey and Triebel-Lizorkin spaces boundedness for the commutator.

\section{Theorems and Lemmas}

We shall prove the following theorems.

Theorem 1. Let $T^{\delta}$ be the integral operator as Definition 1, the sequence $\left\{C_{k}\right\} \in l^{1}, 0<\beta<1, q^{\prime} \leq s<\infty$ and $b \in \operatorname{Lip}_{\beta}\left(R^{n}\right)$. Then there exists a constant $C>0$ such that, for any $f \in C_{0}^{\infty}\left(R^{n}\right)$ and $\tilde{x} \in R^{n}$,

$$
M^{\#}\left(T_{b}^{\delta}(f)\right)(\tilde{x}) \leq C\|b\|_{L i p_{\beta}}\left(M_{\beta+\delta, s}(f)(\tilde{x})+M_{\beta, s}\left(T^{\delta}(f)\right)(\tilde{x})\right) .
$$


Vol. L (2012) Sharp Maximal Function Estimates and Boundedness for Commutator...101

Theorem 2. Let $T^{\delta}$ be the integral operator as Definition 1, the sequence $\left\{2^{k \beta} C_{k}\right\} \in l^{1}, 0<\beta<1, q^{\prime} \leq s<\infty$ and $b \in \operatorname{Lip}_{\beta}\left(R^{n}\right)$. Then there exists a constant $C>0$ such that, for any $f \in C_{0}^{\infty}\left(R^{n}\right)$ and $\tilde{x} \in R^{n}$,

$\sup _{Q \ni \tilde{x}} \inf _{c} \frac{1}{|Q|^{1+\beta / n}} \int_{Q}\left|T_{b}^{\delta}(f)(x)-c\right| d x \leq C|| b \|_{L_{\text {Lip }}}\left(M_{\delta, s}(f)(\tilde{x})+M_{s}\left(T^{\delta}(f)\right)(\tilde{x})\right)$.

Theorem 3. Let $T^{\delta}$ be the integral operator as Definition 1, the sequence $\left\{k C_{k}\right\} \in l^{1}, q^{\prime} \leq s<\infty$ and $b \in B M O\left(R^{n}\right)$. Then there exists a constant $C>0$ such that, for any $f \in C_{0}^{\infty}\left(R^{n}\right)$ and $\tilde{x} \in R^{n}$,

$$
M^{\#}\left(T_{b}^{\delta}(f)\right)(\tilde{x}) \leq C\|b\|_{B M O}\left(M_{\delta, s}(f)(\tilde{x})+M_{s}\left(T^{\delta}(f)\right)(\tilde{x})\right) .
$$

Theorem 4. Let $T^{\delta}$ be the integral operator as Definition 1, the sequence $\left\{C_{k}\right\} \in l^{1}, 0<\beta<\min (1, n-\delta), q^{\prime}<p<n /(\beta+\delta), 1 / r=1 / p-(\beta+\delta) / n$ and $b \in \operatorname{Lip}_{\beta}\left(R^{n}\right)$. Then $T_{b}^{\delta}$ is bounded from $L^{p}\left(R^{n}\right)$ to $L^{r}\left(R^{n}\right)$.

Theorem 5. Let $T^{\delta}$ be the integral operator as Definition 1, the sequence $\left\{C_{k}\right\} \in l^{1}, 0<D<2^{n}, 0<\beta<\min (1, n-\delta), q^{\prime}<p<n /(\beta+\delta)$, $1 / r=1 / p-(\beta+\delta) / n$ and $b \in \operatorname{Lip}_{\beta}\left(R^{n}\right)$. Then $T_{b}^{\delta}$ is bounded from $L^{p, \varphi}\left(R^{n}\right)$ to $L^{r, \varphi}\left(R^{n}\right)$.

Theorem 6. Let $T^{\delta}$ be the integral operator as Definition 1, the sequence $\left\{2^{k \beta} C_{k}\right\} \in l^{1}, 0<\beta<1, q^{\prime}<p<n / \delta, 1 / r=1 / p-\delta / n$ and $b \in \operatorname{Lip}_{\beta}\left(R^{n}\right)$. Then $T_{b}^{\delta}$ is bounded from $L^{p}\left(R^{n}\right)$ to $\dot{F}_{r}^{\beta, \infty}\left(R^{n}\right)$.

Theorem 7. Let $T^{\delta}$ be the integral operator as Definition 1, the sequence $\left\{k C_{k}\right\} \in l^{1}, q^{\prime}<p<n / \delta, 1 / r=1 / p-\delta / n$ and $b \in B M O\left(R^{n}\right)$. Then $T_{b}^{\delta}$ is bounded from $L^{p}\left(R^{n}\right)$ to $L^{r}\left(R^{n}\right)$.

Theorem 8. Let $T^{\delta}$ be the integral operator as Definition 1, the sequence $\left\{k C_{k}\right\} \in l^{1}, 0<D<2^{n}, q^{\prime}<p<n / \delta, 1 / r=1 / p-\delta / n$ and $b \in B M O\left(R^{n}\right)$. Then $T_{b}^{\delta}$ is bounded from $L^{p, \varphi}\left(R^{n}\right)$ to $L^{r, \varphi}\left(R^{n}\right)$.

To prove the theorems, we need the following lemma.

Lemma 1.(see [1]) Let $T^{\delta}$ be the integral operator as Definition 1. Then $T^{\delta}$ is bounded from $L^{p}\left(R^{n}\right)$ to $L^{r}\left(R^{n}\right)$ for $1<p<n / \delta$ and $1 / r=1 / p-\delta / n$.

Lemma 2.(see [19]). For $0<\beta<1$ and $1<p<\infty$, we have

$$
\begin{gathered}
\|f\|_{\dot{F}_{p}^{\beta, \infty}} \approx \| \sup _{Q \ni \cdot} \frac{1}{|Q|^{1+\beta / n}} \int_{Q}\left|f(x)-f_{Q}\right| d x||_{L^{p}} \approx \\
\| \sup _{Q \ni \cdot c} \inf _{c} \frac{1}{|Q|^{1+\beta / n}} \int_{Q}|f(x)-c| d x||_{L^{p}} .
\end{gathered}
$$

Lemma 3. (see [9]). Let $0<p<\infty$ and $w \in \cup_{1 \leq r<\infty} A_{r}$. Then, for any smooth function $f$ for which the left-hand side is finite,

$$
\int_{R^{n}} M(f)(x)^{p} w(x) d x \leq C \int_{R^{n}} M^{\#}(f)(x)^{p} w(x) d x .
$$


Lemma 4.(see [2]) Suppose that $0<\eta<n, 1<s<p<n / \eta$ and $1 / q=$ $1 / p-\eta / n$. Then

$$
\left\|M_{\eta, s}(f)\right\|_{L^{q}} \leq C\|f\|_{L^{p}} .
$$

Lemma 5. Let $1<p<\infty, 0<D<2^{n}$. Then, for any smooth function $f$ for which the left-hand side is finite,

$$
\|M(f)\|_{L^{p, \varphi}} \leq C\left\|M^{\#}(f)\right\|_{L^{p, \varphi}} .
$$

Proof. For any cube $Q=Q\left(x_{0}, d\right)$ in $R^{n}$, we know $M\left(\chi_{Q}\right) \in A_{1}$ for any cube $Q=Q(x, d)$ by [5]. Noticing that $M\left(\chi_{Q}\right) \leq 1$ and $M\left(\chi_{Q}\right)(x) \leq d^{n} /(\mid x-$ $\left.x_{0} \mid-d\right)^{n}$ if $x \in Q^{c}$, by Lemma 3, we have, for $f \in L^{p, \varphi}\left(R^{n}\right)$,

$$
\begin{aligned}
& \int_{Q} M(f)(x)^{p} d x=\int_{R^{n}} M(f)(x)^{p} \chi_{Q}(x) d x \\
\leq & \int_{R^{n}} M(f)(x)^{p} M\left(\chi_{Q}\right)(x) d x \\
\leq & \left.C \int_{R^{n}} M^{\#}(f)(x)\right|^{p} M\left(\chi_{Q}\right)(x) d x \\
= & C\left(\int_{Q} M^{\#}(f)(x)^{p} M\left(\chi_{Q}\right)(x) d x+\sum_{k=0}^{\infty} \int_{2^{k+1} Q \backslash 2^{k} Q} M^{\#}(f)(x)^{p} M\left(\chi_{Q}\right)(x) d x\right) \\
\leq & C\left(\int_{Q} M^{\#}(f)(x)^{p} d x+\sum_{k=0}^{\infty} \int_{2^{k+1} Q \backslash 2^{k} Q} M^{\#}(f)(x)^{p} \frac{|Q|}{\left|2^{k+1} Q\right|} d x\right) \\
\leq & C\left(\int_{Q} M^{\#}(f)(x)^{p} d x+\sum_{k=0}^{\infty} \int_{2^{k+1} Q} M^{\#}(f)(x)^{p} 2^{-k n} d y\right) \\
\leq & C\left\|M^{\#}(f)\right\|_{L^{p, \varphi}}^{p} \sum_{k=0}^{\infty} 2^{-k n} \varphi\left(2^{k+1} d\right) \\
\leq & C\left\|M^{\#}(f)\right\|_{L^{p, \varphi}}^{p} \sum_{k=0}^{\infty}\left(2^{-n} D\right)^{k} \varphi(d) \\
\leq & C\left\|M^{\#}(f)\right\|_{L^{p, \varphi}}^{p} \varphi(d),
\end{aligned}
$$

thus

$$
\left(\frac{1}{\varphi(d)} \int_{Q} M(f)(x)^{p} d x\right)^{1 / p} \leq C\left(\frac{1}{\varphi(d)} \int_{Q} M^{\#}(f)(x)^{p} d x\right)^{1 / p}
$$

and

$$
\|M(f)\|_{L^{p, \varphi}} \leq C\left\|M^{\#}(f)\right\|_{L^{p, \varphi}} .
$$


Vol. L (2012) Sharp Maximal Function Estimates and Boundedness for Commutator...103

This finishes the proof.

Lemma 6. Let $T^{\delta}$ be the integral operator as Definition 1, $0<D<2^{n}$, $1<p<n / \delta$ and $1 / r=1 / p-\delta / n$. Then

$$
\left\|T^{\delta}(f)\right\|_{L^{r, \varphi}} \leq C\|f\|_{L^{p, \varphi}} .
$$

Lemma 7. Let $0<D<2^{n}, 1 \leq s<p<n / \eta$ and $1 / r=1 / p-\eta / n$. Then

$$
\left\|M_{\eta, s}(f)\right\|_{L^{r, \varphi}} \leq C\|f\|_{L^{p, \varphi}} \text {. }
$$

The proofs of two Lemmas are similar to that of Lemma 5 by Lemma 1 and 4 , we omit the details.

\section{Proofs of Theorems}

Proof of Theorem 1. It suffices to prove for $f \in C_{0}^{\infty}\left(R^{n}\right)$ and some constant $C_{0}$, the following inequality holds:

$$
\frac{1}{|Q|} \int_{Q}\left|T_{b}^{\delta}(f)(x)-C_{0}\right| d x \leq\left. C|| b\right|_{L i p_{\beta}}\left(M_{\beta+\delta, s}(f)(\tilde{x})+M_{\beta, s}\left(T^{\delta}(f)\right)(\tilde{x})\right) .
$$

Fix a cube $Q=Q\left(x_{0}, d\right)$ and $\tilde{x} \in Q$. Write, for $f_{1}=f \chi_{2 Q}$ and $f_{2}=f \chi_{(2 Q)^{c}}$, $F_{b}^{\delta, t}(f)(x)=\left(b(x)-b_{2 Q}\right) F^{\delta, t}(f)(x)-F^{\delta, t}\left(\left(b-b_{2 Q}\right) f_{1}\right)(x)-F^{\delta, t}\left(\left(b-b_{2 Q}\right) f_{2}\right)(x)$.

Then

$$
\begin{aligned}
& \frac{1}{|Q|} \int_{Q}\left\|F_{b}^{\delta, t}(f)(x)-F^{\delta, t}\left(\left(b_{2 Q}-b\right) f_{2}\right)\left(x_{0}\right)\right\| d x \\
\leq & \frac{1}{|Q|} \int_{Q}\left\|\left(b(x)-b_{2 Q}\right) F^{\delta, t}(f)(x)\right\| d x+\frac{1}{|Q|} \int_{Q}\left\|F^{\delta, t}\left(\left(b-b_{2 Q}\right) f_{1}\right)(x)\right\| d x \\
& +\frac{1}{|Q|} \int_{Q}\left\|F^{\delta, t}\left(\left(b-b_{2 Q}\right) f_{2}\right)(x)-F^{\delta, t}\left(\left(b-b_{2 Q}\right) f_{2}\right)\left(x_{0}\right)\right\| d x \\
= & I_{1}+I_{2}+I_{3} .
\end{aligned}
$$

For $I_{1}$, by Hölder's inequality, we obtain

$$
\begin{aligned}
I_{1} & \leq \frac{1}{|Q|} \sup _{x \in 2 Q}\left|b(x)-b_{2 Q}\right| \int_{Q}\left|T^{\delta}(f)(x)\right| d x \\
& \leq \frac{1}{|Q|} \sup _{x \in 2 Q}\left|b(x)-b_{2 Q}\right||Q|^{1-1 / s}\left(\int_{Q}\left|T^{\delta}(f)(x)\right|^{s} d x\right)^{1 / s} \\
& \leq C|| b \|_{\text {Lip }_{\beta}}|Q|^{-1 / s}|2 Q|^{\beta / n}|Q|^{1 / s-\beta / n}\left(\frac{1}{|Q|^{1-s \beta / n}} \int_{Q}\left|T^{\delta}(f)(x)\right|^{s} d x\right)^{1 / s} \\
& \leq C\|b\|_{\text {Lip }_{\beta}} M_{\beta, s}\left(T^{\delta}(f)\right)(\tilde{x}) .
\end{aligned}
$$


For $I_{2}$, choose $1<r<\infty$ such that $1 / r=1 / s-\delta / n$, by $\left(L^{s}, L^{r}\right)$-boundedness of $T^{\delta}$ (see Lemma 1), we get

$$
\begin{aligned}
I_{2} & \leq\left(\frac{1}{|Q|} \int_{R^{n}}\left|T^{\delta}\left(\left(b-b_{2 Q}\right) f_{1}\right)(x)\right|^{r} d x\right)^{1 / r} \\
& \leq C|Q|^{-1 / r}\left(\int_{R^{n}}\left|\left(b(x)-b_{2 Q}\right) f_{1}(x)\right|^{s} d x\right)^{1 / s} \\
& \leq C|Q|^{-1 / r}\|b\|_{L i p_{\beta}}|2 Q|^{\beta / n}|2 Q|^{1 / s-(\beta+\delta) / n}\left(\frac{1}{|2 Q|^{1-s(\beta+\delta) / n}} \int_{2 Q}|f(x)|^{s} d x\right)^{1 / s} \\
& \leq C|| b \|_{L_{\text {Lip }}} M_{\beta+\delta, s}(f)(\tilde{x}) .
\end{aligned}
$$

For $I_{3}$, recalling that $s>q^{\prime}$, we have

$$
\begin{aligned}
& I_{3} \leq \frac{1}{|Q|} \int_{Q} \int_{(2 Q)^{c}}\left|b(y)-b_{2 Q} \| f(y)\right||| F^{\delta, t}(x, y)-F^{\delta, t}\left(x_{0}, y\right)|| d y d x \\
& \leq \frac{1}{|Q|} \int_{Q} \sum_{k=1}^{\infty} \int_{2^{k} d \leq\left|y-x_{0}\right|<2^{k+1} d}\left\|F^{\delta, t}(x, y)-F^{\delta, t}\left(x_{0}, y\right)\right\| \\
& \times\left|b(y)-b_{2^{k+1} Q}\right||f(y)| d y d x \\
& +\frac{1}{|Q|} \int_{Q} \sum_{k=1}^{\infty} \int_{2^{k} d \leq\left|y-x_{0}\right|<2^{k+1} d}\left\|F^{\delta, t}(x, y)-F^{\delta, t}\left(x_{0}, y\right)\right\| \\
& \times\left|b_{2^{k+1} Q}-b_{2 Q}\right||f(y)| d y d x \\
& \leq \frac{C}{|Q|} \int_{Q} \sum_{k=1}^{\infty}\left(\int_{2^{k} d \leq\left|y-x_{0}\right|<2^{k+1} d}\left\|F^{\delta, t}(x, y)-F^{\delta, t}\left(x_{0}, y\right)\right\|^{q} d y\right)^{1 / q} \\
& \times \sup _{y \in 2^{k+1} Q}\left|b(y)-b_{2^{k+1} Q}\right|\left(\int_{2^{k+1} Q}|f(y)|^{q^{\prime}} d y\right)^{1 / q^{\prime}} d x \\
& +\frac{C}{|Q|} \int_{Q} \sum_{k=1}^{\infty}\left|b_{2^{k+1} Q}-b_{2 Q}\right|\left(\int_{2^{k} d \leq\left|y-x_{0}\right|<2^{k+1} d} \| F^{\delta, t}(x, y)-F^{\delta, t}\left(x_{0}, y\right) \mid{ }^{q} d y\right)^{1 / q} \\
& \times\left(\int_{2^{k+1} Q}|f(y)|^{q^{\prime}} d y\right)^{1 / q^{\prime}} d x
\end{aligned}
$$


Vol. L (2012) Sharp Maximal Function Estimates and Boundedness for Commutator...105

$$
\begin{aligned}
\leq & \left.C \sum_{k=1}^{\infty} C_{k}\left(2^{k} d\right)^{-n / q^{\prime}+\delta}\left|2^{k+1} Q\right|^{\beta / n}|| b\right|_{L_{i p_{\beta}}}\left|2^{k+1} Q\right|^{1 / q^{\prime}-1 / s}\left|2^{k+1} Q\right|^{1 / s-(\beta+\delta) / n} \\
& \times\left(\frac{1}{\left|2^{k+1} Q\right|^{1-s(\beta+\delta) / n}} \int_{2^{k+1} Q}|f(y)|^{s} d y\right)^{1 / s} \\
& +C \sum_{k=1}^{\infty}|| b \|_{L_{i p_{\beta}}}\left|2^{k} Q\right|^{\beta / n} C_{k}\left(2^{k} d\right)^{-n / q^{\prime} \delta}\left|2^{k+1} Q\right|^{1 / q^{\prime}-1 / s}\left|2^{k+1} Q\right|^{1 / s-(\beta+\delta) / n} \\
& \times\left(\frac{1}{\left|2^{k+1} Q\right|^{1-s(\beta+\delta) / n}} \int_{2^{k+1} Q}|f(y)|^{s} d y\right)^{1 / s} \\
\leq & C\|b\|_{\operatorname{Lip}_{\beta}} M_{\beta+\delta, s}(f)(\tilde{x}) \sum_{k=1}^{\infty} C_{k} \\
\leq & C\|b\|_{\operatorname{Lip}_{\beta}} M_{\beta+\delta, s}(f)(\tilde{x}) .
\end{aligned}
$$

These complete the proof of Theorem 1.

Proof of Theorem 2. It suffices to prove for $f \in C_{0}^{\infty}\left(R^{n}\right)$ and some constant $C_{0}$, the following inequality holds:

$$
\frac{1}{|Q|^{1+\beta / n}} \int_{Q}\left|T_{b}^{\delta}(f)(x)-C_{0}\right| d x \leq\left. C|| b\right|_{L_{\text {Lip }}}\left(M_{\delta, s}(f)(\tilde{x})+M_{s}\left(T^{\delta}(f)\right)(\tilde{x})\right) .
$$

Fix a cube $Q=Q\left(x_{0}, d\right)$ and $\tilde{x} \in Q$. Write, for $f_{1}=f \chi_{2 Q}$ and $f_{2}=f \chi_{(2 Q)^{c}}$, $F_{b}^{\delta, t}(f)(x)=\left(b(x)-b_{2 Q}\right) F^{\delta, t}(f)(x)-F^{\delta, t}\left(\left(b-b_{2 Q}\right) f_{1}\right)(x)-F_{b}^{\delta, t}\left(\left(b-b_{2 Q}\right) f_{2}\right)(x)$.

Then

$$
\begin{aligned}
& \frac{1}{|Q|^{1+\beta / n}} \int_{Q}\left\|F_{b}^{\delta, t}(f)(x)-F^{\delta, t}\left(\left(b_{2 Q}-b\right) f_{2}\right)\left(x_{0}\right)\right\| d x \\
\leq & \frac{1}{|Q|^{1+\beta / n}} \int_{Q}\left\|\left(b(x)-b_{2 Q}\right) F^{\delta, t}(f)(x)\right\| d x \\
& +\frac{1}{|Q|^{1+\beta / n}} \int_{Q}\left\|F^{\delta, t}\left(\left(b-b_{2 Q}\right) f_{1}\right)(x)\right\| d x \\
& +\frac{1}{|Q|^{1+\beta / n}} \int_{Q}\left\|F^{\delta, t}\left(\left(b-b_{2 Q}\right) f_{2}\right)(x)-F^{\delta, t}\left(\left(b-b_{2 Q}\right) f_{2}\right)\left(x_{0}\right)\right\| d x \\
= & I I_{1}+I I_{2}+I I_{3} .
\end{aligned}
$$

By using the same argument as in the proof of Theorem 1, we get, for 
$1<r<\infty$ with $1 / r=1 / s-\delta / n$,

$$
\begin{aligned}
I I_{1} & \leq \frac{C}{|Q|^{1+\beta / n}} \sup _{x \in 2 Q}\left|b(x)-b_{2 Q}\right||Q|^{1-1 / s}\left(\int_{Q}\left|T^{\delta}(f)(x)\right|^{s} d x\right)^{1 / s} \\
& \leq C|Q|^{-1-\beta / n}|| b \|_{L_{i p_{\beta}}}|2 Q|^{\beta / n}|Q|^{1-1 / s}|Q|^{1 / s}\left(\frac{1}{|Q|} \int_{Q}\left|T^{\delta}(f)(x)\right|^{s} d x\right)^{1 / s} \\
& \leq C|| b \|_{L_{\text {in }} \beta} M_{s}\left(T^{\delta}(f)\right)(\tilde{x}),
\end{aligned}
$$

$$
\begin{aligned}
I I_{2} & \leq \frac{1}{|Q|^{1+\beta / n}}|Q|^{1-1 / r}\left(\int_{R^{n}}\left|T^{\delta}\left(\left(b-b_{2 Q}\right) f_{1}\right)(x)\right|^{r} d x\right)^{1 / r} \\
& \leq \frac{C}{|Q|^{1+\beta / n}}|Q|^{1-1 / r}\left(\int_{R^{n}}\left|\left(b(x)-b_{2 Q}\right) f_{1}(x)\right|^{s} d x\right)^{1 / s} \\
& \leq\left.\frac{C}{|Q|^{1+\beta / n}}|Q|^{1-1 / r}|| b\right|_{L_{i p_{\beta}}}|2 Q|^{\beta / n}|2 Q|^{1 / s-\delta / n}\left(\frac{1}{|2 Q|^{1-s \delta / n}} \int_{2 Q}|f(x)|^{s} d x\right)^{1 / s} \\
& \leq\left. C|| b\right|_{L_{\text {Lip }}} M_{\delta, s}(f)(\tilde{x}),
\end{aligned}
$$

$$
\begin{aligned}
I I_{3} \leq & \frac{1}{|Q|^{1+\beta / n}} \int_{Q} \int_{(2 Q)^{c}}\left|b(y)-b_{2 Q}\right||f(y)||| F^{\delta, t}(x, y)-F^{\delta, t}\left(x_{0}, y\right) \| d y d x \\
\leq & \frac{1}{|Q|^{1+\beta / n}} \int_{Q} \sum_{k=1}^{\infty} \int_{2^{k} d \leq\left|y-x_{0}\right|<2^{k+1} d} \| F^{\delta, t}(x, y)-F^{\delta, t}\left(x_{0}, y\right)|| \\
& +\frac{1}{|Q|^{1+\beta / n}} \int_{Q} \sum_{k=1}^{\infty} \int_{2^{k} d \leq\left|y-x_{0}\right|<2^{k+1} d}|| F^{\delta, t}(x, y)-F^{\delta, t}\left(x_{0}, y\right) \| \\
\leq & \frac{C\left|b_{2^{k+1} Q}-b_{2 Q}\right||f(y)| d y d x}{|Q|^{1+\beta / n}} \int_{Q} \sum_{k=1}^{\infty}\left(\int_{2^{k} d \leq\left|y-x_{0}\right|<2^{k+1} d}|| F^{\delta, t}(x, y)-F^{\delta, t}\left(x_{0}, y\right) \mid{ }^{q} d y\right)^{1 / q} \\
& \times \sup _{y \in 2^{k+1} Q}\left|b(y)-b_{2^{k+1} Q}\right|\left(\int_{2^{k+1} Q}|f(y)|^{q^{\prime}} d y\right)^{1 / q^{\prime}} d x
\end{aligned}
$$


Vol. L (2012) Sharp Maximal Function Estimates and Boundedness for Commutator...107

$$
\begin{aligned}
& +\frac{C}{|Q|^{1+\beta / n}} \int_{Q} \sum_{k=1}^{\infty}\left|b_{2^{k+1} Q}-b_{2 Q}\right| \\
& \times\left(\int_{2^{k} d \leq\left|y-x_{0}\right|<2^{k+1} d}|| F^{\delta, t}(x, y)-F^{\delta, t}\left(x_{0}, y\right)||^{q} d y\right)^{1 / q} \\
& \times\left(\int_{2^{k+1} Q}|f(y)|^{q^{\prime}} d y\right)^{1 / q^{\prime}} d x \\
& \leq \quad C|Q|^{-\beta / n} \sum_{k=1}^{\infty} C_{k}\left(2^{k} d\right)^{-n / q^{\prime}+\delta}\left|2^{k+1} Q\right|^{\beta / n}|| b||_{L i p_{\beta}}\left|2^{k+1} Q\right|^{1 / q^{\prime}-\delta / n} \\
& \quad \times\left(\frac{1}{\left|2^{k+1} Q\right|^{1-s \delta / n}} \int_{2^{k+1} Q}|f(y)|^{s} d y\right)^{1 / s} \\
& +C|Q|^{-\beta / n} \sum_{k=1}^{\infty}|| b||_{L i p_{\beta}}\left|2^{k} Q\right|^{\beta / n} C_{k}\left(2^{k} d\right)^{-n / q^{\prime}+\delta}\left|2^{k+1} Q\right|^{1 / q^{\prime}-\delta / n} \\
& \quad \times\left(\frac{1}{\left|2^{k+1} Q\right|^{1-s \delta / n}} \int_{2^{k+1} Q}|f(x)|^{s} d x\right)^{1 / s} \\
& \leq \quad C|| b||_{L i p_{\beta}} M_{\delta, s}(f)(\tilde{x}) \sum_{k=1}^{\infty} 2^{k \beta} C_{k} \\
& \leq C|| b||_{L i p_{\beta}} M_{\delta, s}(f)(\tilde{x}) .
\end{aligned}
$$

This completes the proof of Theorem 2 .

Proof of Theorem 3. It suffices to prove for $f \in C_{0}^{\infty}\left(R^{n}\right)$ and some constant $C_{0}$, the following inequality holds:

$$
\frac{1}{|Q|} \int_{Q}\left|T_{b}^{\delta}(f)(x)-C_{0}\right| d x \leq C|| b \|_{B M O}\left(M_{\delta, s}(f)(\tilde{x})+M_{s}\left(T^{\delta}(f)\right)(\tilde{x})\right) .
$$

Fix a cube $Q=Q\left(x_{0}, d\right)$ and $\tilde{x} \in Q$. Write, for $f_{1}=f \chi_{2 Q}$ and $f_{2}=f \chi_{(2 Q)^{c}}$, $F_{b}^{\delta, t}(f)(x)=\left(b(x)-b_{2 Q}\right) F^{\delta, t}(f)(x)-F^{\delta, t}\left(\left(b-b_{2 Q}\right) f_{1}\right)(x)-F^{\delta, t}\left(\left(b-b_{2 Q}\right) f_{2}\right)(x)$.

Then

$$
\begin{aligned}
& \frac{1}{|Q|} \int_{Q}\left\|F_{b}^{\delta, t}(f)(x)-F^{\delta, t}\left(\left(b_{2 Q}-b\right) f_{2}\right)\left(x_{0}\right)\right\| d x \\
\leq & \frac{1}{|Q|} \int_{Q}\left\|\left(b(x)-b_{2 Q}\right) F^{\delta, t}(f)(x)\right\| d x+\frac{1}{|Q|} \int_{Q}\left\|F^{\delta, t}\left(\left(b-b_{2 Q}\right) f_{1}\right)(x)\right\| d x \\
& +\frac{1}{|Q|} \int_{Q}\left\|F^{\delta, t}\left(\left(b-b_{2 Q}\right) f_{2}\right)(x)-F^{\delta, t}\left(\left(b-b_{2 Q}\right) f_{2}\right)\left(x_{0}\right)\right\| d x \\
= & I I I_{1}+I I I_{2}+I I I_{3} .
\end{aligned}
$$


For $I I I_{1}$, by Hölder's inequality, we get

$$
\begin{aligned}
I I I_{1} & \leq\left(\frac{1}{|Q|} \int_{Q}\left|b(x)-b_{2 Q}\right|^{s^{\prime}} d x\right)^{1 / s^{\prime}}\left(\frac{1}{|Q|} \int_{Q}\left|T^{\delta}(f)(x)\right|^{s} d x\right)^{1 / s} \\
& \leq C\|b\|_{B M O} M_{s}\left(T^{\delta}(f)\right)(\tilde{x}) .
\end{aligned}
$$

For $I I I_{2}$, choose $1<p<s$ such that $1 / r=1 / p-\delta / n$, by Hölder's inequality and $\left(L^{p}, L^{r}\right)$-boundedness of $T_{\delta}$, we obtain

$$
\begin{aligned}
& I I I_{2} \leq\left(\frac{1}{|Q|} \int_{R^{n}}\left|T^{\delta}\left(\left(b-b_{2 Q}\right) f_{1}\right)(x)\right|^{r} d x\right)^{1 / r} \\
& \leq C|Q|^{-1 / r}\left(\int_{R^{n}}\left|\left(b-b_{2 Q}\right) f_{1}(x)\right|^{p} d x\right)^{1 / p} \\
& \leq C|Q|^{-1 / r}\left(\int_{2 Q}\left|b(x)-b_{2 Q}\right|^{s p /(s-p)} d x\right)^{(s-p) / s p}\left(\int_{2 Q}|f(x)|^{s} d x\right)^{1 / s} \\
& \leq C|Q|^{-1 / r}|2 Q|^{(s-p) / s p}\left(\frac{1}{|2 Q|} \int_{2 Q}\left|b(x)-b_{2 Q}\right|^{s p /(s-p)} d x\right)^{(s-p) / s p} \\
& \times|2 Q|^{1 / s-\delta / n}\left(\frac{1}{|2 Q|^{1-s \delta / n}} \int_{2 Q}|f(x)|^{s} d x\right)^{1 / s} \\
& \leq C|| b \|_{B M O}|Q|^{-1 / r+1 / p-\delta / n}\left(\frac{1}{|2 Q|^{1-s \delta / n}} \int_{2 Q}|f(x)|^{s} d x\right)^{1 / s} \\
& \leq C\|b\|_{B M O} M_{\delta, s}(f)(\tilde{x}) \text {. }
\end{aligned}
$$

For $I_{I} I_{3}$, recalling that $s>q^{\prime}$, taking $1<p<\infty$ with $1 / p+1 / q+1 / s=1$, by Hölder's inequality, we obtain

$$
\begin{aligned}
& I I I_{3} \leq \frac{1}{|Q|} \int_{Q} \sum_{k=1}^{\infty} \int_{2^{k} d \leq\left|y-x_{0}\right|<2^{k+1} d}|| F^{\delta, t}(x, y)-F^{\delta, t}\left(x_{0}, y\right) \| \\
& \times\left|b(y)-b_{2 Q}\right||f(y)| d y d x \\
& \leq \frac{1}{|Q|} \int_{Q} \sum_{k=1}^{\infty}\left(\int_{2^{k} d \leq\left|y-x_{0}\right|<2^{k+1} d} \| F^{\delta, t}(x, y)-F^{\delta, t}\left(x_{0}, y\right) \mid{ }^{q} d y\right)^{1 / q} \\
& \times\left(\int_{2^{k+1} Q}\left|b(x)-b_{2 Q}\right|^{p} d x\right)^{1 / p}\left(\int_{2^{k+1} Q}|f(y)|^{s} d y\right)^{1 / s} d x
\end{aligned}
$$


Vol. L (2012) Sharp Maximal Function Estimates and Boundedness for Commutator...109

$$
\begin{gathered}
\leq C \sum_{k=1}^{\infty} C_{k}\left(2^{k} d\right)^{-n / q^{\prime}+\delta} k\left(2^{k} d\right)^{n / p}\|b\|_{B M O}\left(2^{k} d\right)^{n(1 / s-\delta / n)} \\
\times\left(\frac{1}{\left|2^{k+1} Q\right|^{1-s \delta / n}} \int_{2^{k+1} Q}|f(y)|^{s} d y\right)^{1 / s} \\
\leq C\|b\|_{B M O} \sum_{k=1}^{\infty} k C_{k}\left(2^{k} d\right)^{n(-1+1 / q+1 / p+1 / s)}\left(\frac{1}{\left|2^{k+1} Q\right|^{1-s \delta / n}} \int_{2^{k+1} Q}|f(y)|^{s} d y\right)^{1 / s} \\
\leq C\|b\|_{B M O} M_{\delta, s}(f)(\tilde{x}) \sum_{k=1}^{\infty} k C_{k} \\
\leq C\|b\|_{B M O} M_{\delta, s}(f)(\tilde{x}) .
\end{gathered}
$$

This completes the proof of Theorem 3 .

Proof of Theorem 4. Choose $q^{\prime}<s<p$ in Theorem 1 and let $1 / t=$ $1 / p-\delta / n$, then $1 / r=1 / t-\beta / n$, thus, we have, by Lemma 1,3 and 4 ,

$$
\begin{aligned}
& \left\|T_{b}^{\delta}(f)\right\|_{L^{r}} \leq\left\|M\left(T_{b}^{\delta}(f)\right)\right\|_{L^{r}} \leq C\left\|M^{\#}\left(T_{b}^{\delta}(f)\right)\right\|_{L^{r}} \\
\leq & C\|b\|_{L_{i p}}\left(\left\|M_{\beta, s}\left(T^{\delta}(f)\right)\right\|_{L^{r}}+\left\|M_{\beta+\delta, s}(f)\right\|_{L^{r}}\right) \\
\leq & C\|b\|_{L_{i p_{\beta}}}\left(\left\|T^{\delta}(f)\right\|_{L^{t}}+\|f\|_{L^{p}}\right) \\
\leq & C\|b\|_{L_{i p_{\beta}}}\|f\|_{L^{p}} .
\end{aligned}
$$

This completes the proof of Theorem 4 .

Proof of Theorem 5. Choose $q^{\prime}<s<p$ in Theorem 1 and let $1 / t=$ $1 / p-\delta / n$, then $1 / r=1 / t-\beta / n$, thus, we have, by Lemma $5-7$,

$$
\begin{aligned}
& \left\|T_{b}^{\delta}(f)\right\|_{L^{r, \varphi}} \leq\left\|M\left(T_{b}^{\delta}(f)\right)\right\|_{L^{r, \varphi}} \leq C\left\|M^{\#}\left(T_{b}^{\delta}(f)\right)\right\|_{L^{r, \varphi}} \\
\leq & C\|b\|_{L_{i p_{\beta}}}\left(\left\|M_{\beta, s}\left(T_{b}^{\delta}(f)\right)\right\|_{L^{r, \varphi}}+\left\|M_{\beta+\delta, s}(f)\right\|_{L^{r, \varphi}}\right) \\
\leq & C\|b\|_{L_{i p_{\beta}}}\left(\left\|T^{\delta}(f)\right\|_{L^{t, \varphi}}+\|f\|_{L^{p, \varphi}}\right) \\
\leq & C\|b\|_{\text {Lip }_{\beta}}\|f\|_{L^{p, \varphi}} .
\end{aligned}
$$

This completes the proof of Theorem 5 .

Proof Theorem 6. Choose $q^{\prime}<s<p$ in Theorem 2. By using Lemma 2, we obtain

$$
\begin{aligned}
\left\|T_{b}^{\delta}(f)\right\|_{\dot{F}_{r}^{\beta, \infty}} & \leq C\left\|\sup _{Q \ni \cdot} \frac{1}{|Q|^{1+\beta / n}} \int_{Q}\left|T_{b}^{\delta}(f)(x)-T^{\delta}\left(\left(b_{2 Q}-b\right) f_{2}\right)\left(x_{0}\right)\right| d x\right\|_{L^{r}} \\
& \leq C\|b\|_{L_{i p_{\beta}}}\left(\left\|M_{s}\left(T^{\delta}(f)\right)\right\|_{L^{r}}+\left\|M_{\delta, s}(f)\right\|_{L^{r}}\right) \\
& \leq C\|b\|_{L_{i p_{\beta}}}\left(\left\|T^{\delta}(f)\right\|_{L^{r}}+\|f\|_{L^{p}}\right) \\
& \leq C\|b\|_{L_{p_{\beta}}}\|f\|_{L^{p}} .
\end{aligned}
$$


This completes the proof of Theorem 6 .

Proof of Theorem 7. Choose $q^{\prime} \leq s<p$ in Theorem 3 and similar to the proof of Theorem 4, we have

$$
\begin{aligned}
& \left\|T_{b}^{\delta}(f)\right\|_{L^{r}} \leq\left\|M\left(T_{b}^{\delta}(f)\right)\right\|_{L^{r}} \leq C\left\|M^{\#}\left(T_{b}^{\delta}(f)\right)\right\|_{L^{r}} \\
\leq & C\|b\|_{B M O}\left(\left\|M_{s}\left(T^{\delta}(f)\right)\right\|_{L^{r}}+\left\|M_{\delta, s}(f)\right\|_{L^{r}}\right) \\
\leq & C\|b\|_{B M O}\left(\left\|T^{\delta}(f)\right\|_{L^{r}}+\|f\|_{L^{p}}\right) \\
\leq & C\|b\|_{B M O}\|f\|_{L^{p}} .
\end{aligned}
$$

This completes the proof of the theorem.

Proof of Theorem 8. Choose $q^{\prime} \leq s<p$ in Theorem 3 and similar to the proof of Theorem 5, we have

$$
\begin{aligned}
& \left\|T_{b}^{\delta}(f)\right\|_{L^{r, \varphi}} \leq\left\|M\left(T_{b}^{\delta}(f)\right)\right\|_{L^{r, \varphi}} \leq C\left\|M^{\#}\left(T_{b}^{\delta}(f)\right)\right\|_{L^{r, \varphi}} \\
\leq & C\|b\|_{B M O}\left(\left\|M_{s}\left(T^{\delta}(f)\right)\right\|_{L^{r, \varphi}}+\left\|M_{\delta, s}(f)\right\|_{L^{r, \varphi}}\right) \\
\leq & C\|b\|_{B M O}\left(\left\|T^{\delta}(f)\right\|_{L^{r, \varphi}}+\|f\|_{L^{p, \varphi}}\right) \\
\leq & C\|b\|_{B M O}\|f\|_{L^{p, \varphi}} .
\end{aligned}
$$

This completes the proof of the theorem.

\section{Applications}

In this section we shall apply the Theorems 1-8 of the paper to some particular operators such as the Littlewood-Paley operators, Marcinkiewicz operator and Bochner-Riesz operator.

Application 1. Littlewood-Paley operators.

Fixed $0 \leq \delta<n, \varepsilon>0$ and $\mu>(3 n+2) / n$. Let $\psi$ be a fixed function which satisfies:

(1) $\int_{R^{n}} \psi(x) d x=0$,

(2) $|\psi(x)| \leq C(1+|x|)^{-(n+1-\delta)}$,

(3) $|\psi(x+y)-\psi(x)| \leq C|y|^{\varepsilon}(1+|x|)^{-(n+1+\varepsilon-\delta)}$ when $2|y|<|x|$;

We denote $\Gamma(x)=\left\{(y, t) \in R_{+}^{n+1}:|x-y|<t\right\}$ and the characteristic function of $\Gamma(x)$ by $\chi_{\Gamma(x)}$. The Littlewood-Paley commutators are defined by

$$
\begin{gathered}
g_{\psi}^{b}(f)(x)=\left(\int_{0}^{\infty}\left|F_{t}^{b}(f)(x)\right|^{2} \frac{d t}{t}\right)^{1 / 2}, \\
S_{\psi}^{b}(f)(x)=\left[\iint_{\Gamma(x)}\left|F_{t}^{b}(f)(x, y)\right|^{2} \frac{d y d t}{t^{n+1}}\right]^{1 / 2}
\end{gathered}
$$


Vol. L (2012) Sharp Maximal Function Estimates and Boundedness for Commutator...111

and

$$
g_{\mu}^{b}(f)(x)=\left[\iint_{R_{+}^{n+1}}\left(\frac{t}{t+|x-y|}\right)^{n \mu}\left|F_{t}^{b}(f)(x, y)\right|^{2} \frac{d y d t}{t^{n+1}}\right]^{1 / 2},
$$

where

$$
\begin{aligned}
& F_{t}^{b}(f)(x)=\int_{R^{n}}(b(x)-b(y)) \psi_{t}(x-y) f(y) d y, \\
& F_{t}^{b}(f)(x, y)=\int_{R^{n}}(b(x)-b(z)) f(z) \psi_{t}(y-z) d z
\end{aligned}
$$

and $\psi_{t}(x)=t^{-n} \psi(x / t)$ for $t>0$. Set $F_{t}(f)(y)=f * \psi_{t}(y)$. We also define

$$
\begin{gathered}
g_{\psi}(f)(x)=\left(\int_{0}^{\infty}\left|F_{t}(f)(x)\right|^{2} \frac{d t}{t}\right)^{1 / 2}, \\
S_{\psi}(f)(x)=\left(\iint_{\Gamma(x)}\left|F_{t}(f)(y)\right|^{2} \frac{d y d t}{t^{n+1}}\right)^{1 / 2}
\end{gathered}
$$

and

$$
g_{\mu}(f)(x)=\left(\iint_{R_{+}^{n+1}}\left(\frac{t}{t+|x-y|}\right)^{n \mu}\left|F_{t}(f)(y)\right|^{2} \frac{d y d t}{t^{n+1}}\right)^{1 / 2},
$$

which are the Littlewood-Paley operators (see [25]). Let $H$ be the space

$$
H=\left\{h:\|h\|=\left(\int_{0}^{\infty}|h(t)|^{2} d t / t\right)^{1 / 2}<\infty\right\}
$$

or

$$
H=\left\{h:\|h\|=\left(\iint_{R_{+}^{n+1}}|h(y, t)|^{2} d y d t / t^{n+1}\right)^{1 / 2}<\infty\right\},
$$

then, for each fixed $x \in R^{n}, F_{t}^{b}(f)(x)$ and $F_{t}^{b}(f)(x, y)$ may be viewed as the mapping from $[0,+\infty)$ to $H$, and it is clear that

$$
\begin{gathered}
g_{\psi}^{b}(f)(x)=\left\|F_{t}^{b}(f)(x)\right\|, \quad g_{\psi}(f)(x)=\left\|F_{t}(f)(x)\right\|, \\
S_{\psi}^{b}(f)(x)=\left\|\chi_{\Gamma(x)} F_{t}^{b}(f)(x, y)\right\|, \quad S_{\psi}(f)(x)=\left\|\chi_{\Gamma(x)} F_{t}(f)(y)\right\|
\end{gathered}
$$

and

$$
g_{\mu}^{b}(f)(x)=\left\|\left(\frac{t}{t+|x-y|}\right)^{n \mu / 2} F_{t}^{b}(f)(x, y)\right\|
$$




$$
g_{\mu}(f)(x)=\left\|\left(\frac{t}{t+|x-y|}\right)^{n \mu / 2} F_{t}(f)(y)\right\| .
$$

It is easily to see that $g_{\psi}^{b}, S_{\psi}^{b}$ and $g_{\mu}^{b}$ satisfy the conditions of Theorems 1-8(see [13-15]), thus theorems 1-8 hold for $g_{\psi}^{b}, S_{\psi}^{b}$ and $g_{\mu}^{b}$.

Application 2. Marcinkiewicz operators.

Fixed $0 \leq \delta<n, \lambda>\max (1,2 n /(n+2))$ and $0<\gamma \leq 1$. Let $\Omega$ be homogeneous of degree zero on $R^{n}$ with $\int_{S^{n-1}} \Omega\left(x^{\prime}\right) d \sigma\left(x^{\prime}\right)=0$. Assume that $\Omega \in \operatorname{Lip}_{\gamma}\left(S^{n-1}\right)$. The Marcinkiewicz commutators are defined by

$$
\begin{gathered}
\mu_{\Omega}^{b}(f)(x)=\left(\int_{0}^{\infty}\left|F_{t}^{b}(f)(x)\right|^{2} \frac{d t}{t^{3}}\right)^{1 / 2} \\
\mu_{S}^{b}(f)(x)=\left[\iint_{\Gamma(x)}\left|F_{t}^{b}(f)(x, y)\right|^{2} \frac{d y d t}{t^{n+3}}\right]^{1 / 2}
\end{gathered}
$$

and

$$
\mu_{\lambda}^{b}(f)(x)=\left[\iint_{R_{+}^{n+1}}\left(\frac{t}{t+|x-y|}\right)^{n \lambda}\left|F_{t}^{b}(f)(x, y)\right|^{2} \frac{d y d t}{t^{n+3}}\right]^{1 / 2},
$$

where

$$
F_{t}^{b}(f)(x)=\int_{|x-y| \leq t}(b(x)-b(y)) \frac{\Omega(x-y)}{|x-y|^{n-1-\delta}} f(y) d y
$$

and

$$
F_{t}^{b}(f)(x, y)=\int_{|y-z| \leq t}(b(x)-b(z)) \frac{\Omega(y-z)}{|y-z|^{n-1-\delta}} f(z) d z
$$

Set

$$
F_{t}(f)(x)=\int_{|x-y| \leq t} \frac{\Omega(x-y)}{|x-y|^{n-1-\delta}} f(y) d y
$$

We also define

$$
\begin{gathered}
\mu_{\Omega}(f)(x)=\left(\int_{0}^{\infty}\left|F_{t}(f)(x)\right|^{2} \frac{d t}{t^{3}}\right)^{1 / 2} \\
\mu_{S}(f)(x)=\left(\iint_{\Gamma(x)}\left|F_{t}(f)(y)\right|^{2} \frac{d y d t}{t^{n+3}}\right)^{1 / 2}
\end{gathered}
$$

and

$$
\mu_{\lambda}(f)(x)=\left(\iint_{R_{+}^{n+1}}\left(\frac{t}{t+|x-y|}\right)^{n \lambda}\left|F_{t}(f)(y)\right|^{2} \frac{d y d t}{t^{n+3}}\right)^{1 / 2}
$$


Vol. L (2012) Sharp Maximal Function Estimates and Boundedness for Commutator...113

which are the Marcinkiewicz operators(see [26]). Let $H$ be the space

$$
H=\left\{h:\|h\|=\left(\int_{0}^{\infty}|h(t)|^{2} d t / t^{3}\right)^{1 / 2}<\infty\right\}
$$

or

$$
H=\left\{h:\|h\|=\left(\iint_{R_{+}^{n+1}}|h(y, t)|^{2} d y d t / t^{n+3}\right)^{1 / 2}<\infty\right\} .
$$

Then, it is clear that

$$
\begin{gathered}
\mu_{\Omega}^{b}(f)(x)=\left\|F_{t}^{b}(f)(x)\right\|, \quad \mu_{\Omega}(f)(x)=\left\|F_{t}(f)(x)\right\|, \\
\mu_{S}^{b}(f)(x)=\left\|\chi_{\Gamma(x)} F_{t}^{b}(f)(x, y)\right\|, \quad \mu_{S}(f)(x)=\left\|\chi_{\Gamma(x)} F_{t}(f)(y)\right\|
\end{gathered}
$$

and

$$
\begin{gathered}
\mu_{\lambda}^{b}(f)(x)=\left\|\left(\frac{t}{t+|x-y|}\right)^{n \lambda / 2} F_{t}^{b}(f)(x, y)\right\|, \\
\mu_{\lambda}(f)(x)=\left\|\left(\frac{t}{t+|x-y|}\right)^{n \lambda / 2} F_{t}(f)(y)\right\| .
\end{gathered}
$$

It is easily to see that $\mu_{\Omega}^{b}, \mu_{S}^{b}$ and $\mu_{\lambda}^{b}$ satisfy the conditions of Theorems 1-8 (see [13-15][26]), thus Theorems 1-8 hold for $\mu_{\Omega}^{b}, \mu_{S}^{b}$ and $\mu_{\lambda}^{b}$.

Application 3. Bochner-Riesz operator.

Let $\delta>(n-1) / 2, B_{t}^{\delta}\left(f \hat{)}(\xi)=\left(1-t^{2}|\xi|^{2}\right)_{+}^{\delta} \hat{f}(\xi)\right.$ and $B_{t}^{\delta}(z)=t^{-n} B^{\delta}(z / t)$ for $t>0$. Set

$$
F_{\delta, t}^{b}(f)(x)=\int_{R^{n}}(b(x)-b(y)) B_{t}^{\delta}(x-y) f(y) d y,
$$

The maximal Bochner-Riesz commutator is defined by

$$
B_{\delta, *}^{b}(f)(x)=\sup _{t>0}\left|B_{\delta, t}^{b}(f)(x)\right| .
$$

We also define that

$$
B_{\delta, *}(f)(x)=\sup _{t>0}\left|B_{t}^{\delta}(f)(x)\right|
$$

which is the maximal Bochner-Riesz operator(see [16]). Let $H$ be the space $H=\left\{h:|| h||=\sup _{t>0}|h(t)|<\infty\right\}$, then

$$
B_{\delta, *}^{b}(f)(x)=\left\|B_{\delta, t}^{b}(f)(x)\right\|, \quad B_{*}^{\delta}(f)(x)=\left\|B_{t}^{\delta}(f)(x)\right\| .
$$

It is easily to see that $B_{\delta, *}^{b}$ satisfies the conditions of Theorems 1-8 with $\delta=0\left(\right.$ see [13]), thus Theorems 1-8 hold for $B_{\delta, *}^{b}$. 


\section{References}

[1] D. C. Chang, J. F. Li, and J. Xiao, Weighted scale estimates for CalderónZygmund type operators, Contemporary Mathematics, 446, (2007), 61-70.

[2] S. Chanillo, A note on commutators, Indiana Univ. Math. J., 31, (1982), 7-16.

[3] W. G. Chen, Besov estimates for a class of multilinear singular integrals, Acta Math. Sinica, 16, (2000), 613-626.

[4] F. Chiarenza and M. Frasca, Morrey spaces and Hardy-Littlewood maximal function, Rend. Mat., 7, (1987), 273-279.

[5] R. Coifman and R. Rochberg, Another characterization of BMO, Proc. Amer. Math. Soc., 79, (1980), 249-254.

[6] R. R. Coifman, R. Rochberg, and G. Weiss, Fractorization theorems for Hardy spaces in several variables, Ann. of Math., 103, (1976), 611-635.

[7] G. Di FaZio and M. A. Ragusa, Commutators and Morrey spaces, Boll. Un. Mat. Ital., 5-A(7), (1991), 323-332.

[8] G. Di Fazio and M. A. Ragusa, Interior estimates in Morrey spaces for strong solutions to nondivergence form equations with discontinuous coefficients, J. Func. Anal., 112, (1993), 241-256.

[9] J. Garcia-Cuerva and J. L. Rubio de Francia, Weighted norm inequalities and related topics, North-Holland Math., Amsterdam, 16, 1985.

[10] S. Janson, Mean oscillation and commutators of singular integral operators, Ark. Math., 16, (1978), 263-270.

[11] Y. Lin, Sharp maximal function estimates for Calderón-Zygmund type operators and commutators, Acta Math. Scientia, 31(A), (2011), 206-215.

[12] L. Z. Liu, Interior estimates in Morrey spaces for solutions of elliptic equations and weighted boundedness for commutators of singular integral operators, Acta Math. Scientia, 25(B),(1), (2005), 89-94.

[13] L. Z. Liu, The continuity of commutators on Triebel-Lizorkin spaces, Integral Equations and Operator Theory, 49, (2004), 65-76.

[14] L. Z. Liu, Triebel-Lizorkin space estimates for multilinear operators of sublinear operators, Proc. Indian Acad. Sci. (Math. Sci), 113, (2003), 379-393.

[15] L. Z. Liu, Boundedness for multilinear Littlewood-Paley operators on TriebelLizorkin spaces, Methods and Applications of Analysis, 10(4), (2004), 603-614.

[16] S. Z. Lu, Four lectures on real $H^{p}$ spaces, World Scientific, River Edge, NI, 1995.

[17] T. Mizuhara, Boundedness of some classical operators on generalized Morrey spaces, "Harmonic Analysis", Proceedings of a conference held in Sendai, (1990), 183-189.

[18] B. Muckenhoupt and R. L. Wheeden, Weighted norm inequalities for fractional integral, Trans. Amer. Math. Soc., 192, (1974), 261-274.

[19] M. Paluszynski, Characterization of the Besov spaces via the commutator operator of Coifman, Rochberg and Weiss, 44, (1995), 1-17. 
Vol. L (2012) Sharp Maximal Function Estimates and Boundedness for Commutator...115

[20] J. Peetre, On convolution operators leaving $L^{p, \lambda}$-spaces invariant, Ann. Mat. Pura. Appl., 72, (1966), 295-304.

[21] J. Peetre, On the theory of $L^{p, \lambda}$-spaces, J. Func. Anal., 4, (1969), 71-87.

[22] C. Pérez, Endpoint estimate for commutators of singular integral operators, J. Func. Anal., 128, (1995), 163-185.

[23] C. Pérez and R. Trujillo-Gonzalez, Sharp weighted estimates for multilinear commutators, J. London Math. Soc., 65, (2002), 672-692.

[24] E. M. Stein, Harmonic analysis: real variable methods, orthogonality and oscillatory integrals, Princeton Univ. Press, Princeton NJ, 1993.

[25] A. Torchinsky, Real variable methods in harmonic analysis, Pure and Applied Math. 123, Academic Press, New York, 1986.

[26] A. Torchinsky and S. Wang, A note on the Marcinkiewicz integral, Colloq. Math., 60/61, (1990), 235-243.

Guo Sheng

Department of Mathematics

Changsha University of Science and Technology

Changsha 410077, P. R. of China

E-mail: lanzheliu@163.com

Huang Chuangxia

Department of Mathematics

Changsha University of Science and Technology

Changsha 410077, P. R. of China

Liu Lanzhe

Department of Mathematics

Changsha University of Science and Technology

Changsha 410077, P. R. of China

Received: 4.06.2012

Accepted: 19.10.2012 